\title{
LISTE DES PUBLICATIONS
}

\section{CONCERNANT LES TRAVAUX DE LA STATION EXPERIMENTALE OU POUR LESQUELS ONT ETE UTILISES DES MATERIAUX RECOLTES A RICHELIEU}

\author{
(1934-1961)
}

\section{4}

Brumpt (E.). - Titres et travaux scientifiques (1897-1934). Paris, Masson et Cir, éditeurs, in- $4^{\circ}$, p. 1-201, fig. 1-102.

Dollfus (R.-Ph.). - Sur « Taenia $»$ gallinulae P. J. Van Beneden 1858. Ann. de Parasit., XII, 1934, p. 267-272.

Dollfus (R.-Ph.), Callot (J.) et Desportes (C.). - Sur une cercaire du groupe vitrina et sa métacercaire enkystée. Ann. de Parasit., XII, 1934, p. 521-527.

Dollfus (R.-Ph.). - Sur quelques Brachylaemus de la Faune française récoltês. principalement à Richelieu. Ann. de Parasit., XII, 1934, p. 551-575.

Nitzulescu (Virgil) et Dollfus (R.-Ph.). — Présence à Richelieu (Indre-et-Loire) de Phlebotomus perniciosus Newstead. Ann. de Parasit., XII, 1934, p. 69.

\section{5}

Brumpt (E.). - - Au sujet de la prétendue schizogonie régressive des gamètes femelles d'Haemoproteus paddae: présentation de préparations. Bull. Soc. Pathol. Exot., XXVII, n ${ }^{\circ}$ 3, 1935, p. 114-154, fig. 1-8.

Dollfus (R.-Ph.). — Les Distomes des Stylommatophores terrestres (excl. Succineidae). Catalogue par hôtes et résumé des descriptions. Ann. de Parasit., XIII, 1935, p. 176-189, 259-278, 369-385, 445-485, fig. 1-6.

Callot (J.). - Première note sur les parasites de Sauterelles à Richelieu (I.-et-L.). Ann. de Parasit., XIII, 1935, p. 193-202.

Dollfus (R.-Ph.). - Thélohaniose de l'Ecrevisse (Astacus pallipes Lereboullet), à Richelieu (Indre-et-Loire). Bull. Soc. Centrale d'Aquiculture et de Pêche, XLII, $\mathrm{n}^{\circ}$ 10-12, p. 119-122.

Dollfus (R.-Ph.), Callot (J.) et Desportes (C.). - Infestation expérimentale de Strigiformes par un Brachylaemus. Ann. de Parasit., XIII, 1935, p. 12-20.

Dollfus (R.-Ph.). - Sur quelques Brachylaemus de la Faune française récoltés principalement à Richelieu (I.-et-L.) (suite et fin). Ann. de Parasit., XIII, 1935 , p. $52-79$. 
Dollfus (R.-Ph.), Callot (J.) et Desportes (C.). - Distoma isostoma Rudolphi 1819, parasite d'Astacus, est une métacercaire d'Orchipedum. Ann. de Parasit., XIII, 1935, p. 116-132.

\section{6}

Brumpt (E.). - Action des hôtes définitifs sur l'évolution et sur la sélection des sexes de certains helminthes hébergés par eux. Expériences sur des Schistosomes. Ann. de Parasit., XIV, $\mathrm{n}^{\circ}$ 6, 1936, p. 541-551, fig. 1-2.

Brumpt (E.). - Etude expérimentale du Plasmodium gallinaceum, parasite de la poule domestique. Transmission de ce germe par Stegomyia fasciata et Stegomyia albopicta. Ann. de Parasit., XIV, $\mathrm{n}^{\circ} 6,1936$, p. 597-620, fig. 1-10.

Brumpt (E.). - Contribution à l'étude de l'évolution des Paramphistomidés. Paramphistomum cervi et cercaire de Planorbis exustus. Ann. de Parasit., XIV, 1936, p. 552-563.

BRUMPT (E.). - Supplément à la notice sur les Titres et travaux scientifiques du $D^{r}$ Emile Brumpt (mars 1934-novembre 1936). Paris, Masson et $C^{1 e}$, édit., in $4^{\circ}$, p. 1-72, fig. 1-37.

Dollfus (R.-Ph.). - Présence d'un Pseudamphistoma chez la Loutre à Richelieu (Indre-et-Loire). Ann. de Parasit., XIV, 1936, p. 520-22, fig. 1-2.

\section{7}

Callot (J.). - Sur les parasites de Sauterelles à Richelieu (I.-et-L.). II. Ann. de Parasit., XV, 1937, p. 282.

\section{8}

Brumpt (E.) et Desportes (C.). - Hôtes intermédiaires expérimentaux de deux espèces d'Acanthocéphales (Prosthenorchis spirula et $\boldsymbol{P}$. elegans), parasites des Lémuriens et des Singes. Ann. de Parasit., XVI, 1938, n 4, p. 301-304.

Callot (J.). - Sur un Diphyllobothrium de la Loutre. Ann. de Parasit., XVI, 1938 , p. 526-529, fig. 1-2.

Desportes (C.). - Filaria oesophagea Polonio 1859, parasite de la couleuvre d'Italie, est un Dracunculus très voisin de la Filaire de Médine. Ann. de Parasit., XVI, 1938, $\mathrm{n}^{\circ}$ 4, p. 305-326, fig. 1-10.

Dollfus (R.-Ph.). - Cycle évolutif d'un Trématode du genre Coïtocaecum W. Nicoll. Progénèse de la larve métacercaire chez des Amphipodes. C. $R$. Séances Acad. Sciences, CCXVII, 1938, p. 431-433. Errata, p. 480.

FArréras (E.). - Contribution à l'étude de la ponte des Culex. Ann. de Parasit., XVI, 1938 , p. $146-150$.

1939-1940

Callot (J.). - Sur quelques gites et associations larvaires de Moustiques. Ann. de Parasit., XVII, 1939, p. 86-87. 
Desportes (C.) et Harant (H.). - Observations sur la biologie d'un Cératopogonine hématophage, Forcipomyia velox, Winn 1852, piqueur de la grenouille verte. Ann. de Parasit., XVII, 1939, p. 369-374.

LAvier (G.). - Sur une Coccidie atypique de Vipera aspis. Ann. de Parasit., XVII. 1939 , p. $37-44$, fig. $1-2$.

\section{1}

Brumpt (E.). - Mécanisme d'éclosion des Moustiques. Ann. de Parasit., XVIII. 1941, p. $75-94$, fig. 1-7.

Brumpt (E.). - Les Entomophtorées, parasites des Moustiques. Ann. de Parasit., XVIII, 1941, p. 112-144, fig. 1-15.

DESPORTES (C.). - Nouvelles recherches sur la morphologie et sur l'évolution d'Icosiella neglecta (Diesing, 1851), filaire commune de la Grenouille verte. Ann. de Parasit., XVIII, 1941, p. 46-67, fig. 1-6.

Dollfus (R.-Ph.). - Titres et travaux scientifiques. Paris, octobre 1941, 76 p. LANGeron (M.) et GuerRa (P.). - Les secteurs clairs et sombres des colonies de levures. Ann. de Parasit., XVIII, 1941, p. 95-111, pl. IV-IX.

\section{$1942-1943$}

Brumpt (E.). - Comportement du Soya de la variété 23 du Centre national du Soya de Bordeaux, à la Station expérimentale de Richelieu (Indre-etLoire). Rev. intern. Soya, 1943, $\mathrm{n}^{\circ} 21$, p. 147.

Brumpt et Dao Van Ty. - Distribution des biotypes d'Anopheles maculipennis en France. Ann. de Parasit., XIX, $\mathrm{n}^{\circ s} 1-3,1942$, p. 69-73.

BRUMPT (L.-Ch.). - Le traitement des coccidioses des animaux domestiques par la quinacrine ou atébrine. Ann. de Parasit., XIX, n ${ }^{\circ s} 4-6,1943$, p. 97-115.

CALlot (J.). - Sur Culex hortensis et Culex apicalis à Richelieu (I.-et-L.). Ann. de Parasit., XIX, 1942, p. 129-141.

Callot (J.) et DAo VAN Ty. - Localités françaises nouvelles pour Culex (Culex) theileri, Culex (Barraudius) modestus et Culex (Neoculex) impudicus. Ann. de Parasit., XIX, 1942, p. 142-150.

DespoRtes (C.). - Forcipomyia velox Winn. et Sycorax silacea Curtis, vecteurs d'lcosiella neglecta (Diesing), filaire commune de la Grenouille verte. Ann. de Parasit., XIX, 1942, p. 53-68.

Desportes (C.). - Un curieux Nématode, Heligmosomum costellatum (Dujardin 1845). Ann. de Parasit., XIX, 1942, p. 160-167.

\section{4-1945}

BRuMPT (E.). - Recherche sur la sélection, la grande variabilité et le rendement des Tournesols. C. R. Acad. Sc., CCXVIII, 1944, p. 209-211.

BRUMPT (E.). - Curieux mode d'enkystement d'une cercaire (Xiphidiocercaria exocystis n. sp.) de Bythinia tentaculata. Ann. de Parasit., XX, 1944-45, p. 94-96, fig. 1-2. 
BRUMPT (E.). - Recherches biologiques diverses concernant le cycle évolutif du Trématode Opisthioglyphe ranae (Plagiorchiidae). Ann. de Parasit., XX, 1944-1945, p. 209-243, fig. 1-3.

Brumpt (E.). - Cycle évolutif du Trématode Leptophallus nigrovenosus, parasite de la couleuvre à collier (Tropidonotus natrix) et expérimentalement de la vipère (Vipera aspis). Ann. de Parasit., XX, 1944-1945, p. 244-262, fig. 1-13.

Dollfus (R.-Ph.). - Hyperparasitisme et castration parasitaire par un sporozoaire chez un Cestode. C. R. Acad. Sc., CCXVI, n 11, 1943 (paru 1944), p. 270-272, fig. 1 .

SÉGUY (E.). - Insectes ectoparasites (Mallophages, Anoploures, Siphonaptères). Faune de France, Lechevalier, édit., Paris, t. 43, 1944, p. 1-684, fig. 1-957.

\section{5}

CAllot (J.) et DaO VAN TY. - Contribution à l'étude des Moustiques français. Culicidés de Richelieu (I.-et-L.). Ann. de Parasit., XX, 1944-45, p. 43-66, pl. I-II.

Desportes (C.). - La dermatite des nageurs. Ann. de Parasit., XX, 1944-1945, p. 263-278, fig. 1 .

DESPORTES (C.). - Volumineux sarcome hépatique à Cysticercus fasciolaris chez un surmulot. Ann. de Parasit., XX, 1944-1945, p. 288-296.

Dollfus (R.-Ph.) et Callot (J.). - Etudes documentaires sur le genre Metorchis A. Looss 1899. Observations sur des Metorchis récoltés à Richelieu (Indreet-Loire). Ann. de Parasit., XX, $\mathrm{n}^{\circ s} 3-4,1945$, p. 125-159, fig. 1-20.

Dollfus (R.-Ph.) et Desportes (C.). - Sur le genre Rictularia Froelich 1802 (Nématodes Spiruroidea). Ann. de Parasit., XX, 1944-1945, p. 6-34, fig. 1-2.

Langeron (M.). - Précis de Mycologie, 674 p. Paris, 1945, Masson et C $C^{10}$, édit.

\section{6}

Brumpt (E.). - Elevage et alimentation des Cobayes au centre de Richelieu (Indreet-Loire). Ann. de Parasit., XXI, 1946, p 89-92.

BRUMPT (E.). - Essais de culture de 23 variétés de Soja à la Station Universitaire de Richelieu (Indre-et-Loire). C. R. Acad. Sc., CCXXII, 1946, p. 408-410.

BRUMPT (E.). - Second supplément à la notice sur les Titres et travaux scientifiques du $D^{r}$ Emile Brumpt (novembre 1936-janvier 1946). Paris, Masson et $\mathrm{C}^{\mathrm{te}}$, édit., in- $4^{\circ}$, p. 1-8.

Callot (J.). - Un autre Culicoïdes agressif pour l'homme. Ann. de Parasit., XXI, 1946, p. 379.

Cochet (G.). - Résultats statistiques sur la flore mycologique intestinale de quelques rongeurs de la Faune de France. Ann. de Parasit., XXI, 1946, p. 169-176.

Desportes (C.). - Des Filaires dans le tube digestif. Ann. de Parasit., XXI, 1946. p. $138-141$, fig. 1 . 
Desportes (C.). - Sur un nouveau Physaloptère de la Bondrée, Pernis apivorus L. Ann. de Parasit., XXI, 1946, p. 257-262, fig. 1-3.

Dollfus (R.-Ph.). - Sur un Distome du genre Tamerlania K. I. Skrjabin 1924 avec un catalogue des Trématodes des reins d'oiseaux. Ann. de Parasit., XXI, 1946, p. 25-73, fig. 1-17.

Dollfus (R.-Ph.). - Parasites (animaux et végétaux) des Helminthes. Hyperparasites, ennemis et prédateurs des Helminthes parasites et des Helminthes libres. Encyclopédie biologique, Lechevalier, édit., Paris, t. 27, 1946, p. I-VII $+1-482$, fig. 1-373.

1947

Brumpt (E.). - Camille Desportes, $1909-1947$ (Notice nécrologique). Ann. de Parasit., XXII, p. 289-295.

BRumpt (E.) et Chabaud (A.-G.). — L'infestation par les Ixodidés provoque-t-elle une immunité chez l'hôte? Ann. de Parasit., XXII, 1947, p. 348-356.

Callor (J.). - Sur les gîtes larvaires de Culex hortensis et Culex apicalis. Ann. de Parasit., XXII, 1947, p. 81-83.

Callot (J.). - Sur les larves de Culex hortensis Fic. et de Culex deserticola K. Ann. de Parasit., XXII, 1947, p. 480-481.

Callot (J.). - Etude de quelques souches de Culex pipiens (sensu lato) et sur leurs hybrides. Ann. de Parasit., XXII, 1947, p. 380-393.

Desportes (C.). - Une nouvelle espèce de Synhimantus, parasite des Rapaces. Ann. de Parasit., XXII, 1947, p. 164-168, fig. 1-2.

Dollfus (R.-Ph.). - Cœnurose de la cavité abdominale chez un écureuil (Sciurus vulgaris L.) à Richelieu (I.-et-L.). Ann. de Parasit., XXII, 1947, p. 143147 (paru 30 mars 1948), fig. 1-11.

DupuIs (C.). - Caractères sexuels secondaires des larves et nymphes des Hémiptères hétéroptères. La Feuille des Naturalistes, Bull. des N.P., n. s. 2, fasc. 4 , pp. $33-47,2$ fig.

Dupuis (C.). - Formes préimaginales d'Hémiptères Pentatomidae I. : les nymphes des Asopinae: Pinthaeus sanguinipes F. et Arma custos F. Bull. de la Soc. Entomologique de France, 52, $\mathrm{n}^{\circ} 4$, p. 54-57, 2 fig.

DupuIs (C.). - Insectes parasites nouveaux de Palomena prasina L. (Hémiptères Pentatomidés) à Richelieu (I.-et-L.). Ann. de Parasitologie hum. et comp., 21 (1946), $\mathrm{n}^{\circ s} 5-6$, pp. 302-330, 11 fig. (= Contribution I à l'Etude des Phasiinae cimicophages).

\section{8}

Dollfus (R.-Ph.). - Sur les Prosthogoniminae, Trématodes de la bourse de Fabricius des Oiseaux et leur biogéographie. Mém. Mus. Nat. Hist. Nat., Paris, n. s., XXIV, fasc. 1, 1948, p. 1-73, pl. I-IX, fig. 1-36.

Dollfus (R.-Ph.). - Distome énigmatique dans la vésicule biliaire de la tanche commune Tinca tinca (L.) à Richelieu (I.-et-L.). Ann. de Parasit., XXIII, 1948, p. 14-17, fig. 1. 
Dollfus (R.-Ph.). - Sur deux Monostomes (Cyclocoelidae) pourvus d'une ventouse ventrale. Observations sur la classification des Cyclocoeloidea Albert Henry 1923 ; liste de leurs hôtes, répartition géographique. Ann. de Parasit., XXIII, $\mathrm{n}^{\text {os }} 3-4,1948$, p. 129-199, fig. 1-14.

Dupuis (C.). - Notes faunistiques sur quelques Orthoptères français. I: Orthoptères et Dermaptères de Richelieu (I.-et-L.) (1946-1947). Feuille des Naturalistes, Bull. des N.P., n. s. 3, fasc. 3-4, pp. 41-46.

Dupuis (C.). - Id. II : Mantis religiosa L. dans la région parisienne de 1942 à 1947. Feuille des Naturalistes, Bull. des N.P., n. s. 3, fasc. 5, pp. 53-56.

Dupuis (C.). - Remarques sur le mode de spécificité parasitaire des Phasiinae (Diptères Larvaevoridae). Bull. Biol. Fr. Belgique, 82, fasc. 2-3, pp. 130140. (= Contribution V à l'Etude des Phasiinae cimicophages).

Dupuis (C.). - Nouvelles données biologiques et morphologiques sur les Diptères Phasiinae, parasites d'Hémiptères hétéroptères. Ann. de Parasitologie hum. et comp., 22 (1947), $\mathrm{n}^{\circ}$ 3-4, pp. 201-232, 1 fig. (Première partie: Biologie $=$ Contribution III à l'Etude des Phasiinae cimicophages).

Dupuis (C.) - (Même titre; suite: Morphologie, etc...). Ann. de Parasitologie hum. et comp., 22 (1947), $\mathrm{n}^{\circ}$ 5-6, pp. 397-441, fig. 2-36. (= Contribution IV à l'Etude des Phasiinae cimicophages).

Dupuis (C.). - (Même titre, les deux parties réunies). Mémoire présenté à la Faculté des Sciences de l'Université de Paris pour l'obtention du diplôme d'Etudes Supérieures (Sciences Naturelles), 3 juin 1948, $\mathrm{n}^{\circ}$ d'ordre 970, Masson et $\mathrm{C}^{\text {le }}$ édit., 80 p., 36 fig.

\section{9}

Brumpt (E.). Précis de Parasitologie, $6^{\mathrm{e}}$ édit., 2 vol. Paris, Masson et $\mathrm{C}^{\mathrm{te}}$, édit.

Brumpt (E.) et Buttner (A.). - Pouvoir infectieux des métacercaires d'Echinostomidés. Ann. de Parasit., XXIV, 1949, p. 9-15.

BRUMPT (E.) et ButTNer (A.). - Rôle pathogène de certaines cercaires d'Echinostomidés s'enkystant dans les reins des Tétards. Infections mortelles et infections en partie prémunitives. Ann. de Parasit., XXIV, 1949, p. 16-24, fig. 1.

CARAYON (J.). - Caractères distinctifs, répartitions géographiques et habitats des espèces paléarctiques du genre Alloorhynchus Fieb. (Hem. Nabidae). Bull. Soc. Entom. Fr., 54, novembre 1949, pp. 136-142, fig. 1 A-B.

Desportes (C.), Chabaud (A.-G.) et Campana (Y.). - Sur les Gongylonèmes de Muridae et leurs formes larvaires. Ann. de Parasit., XXIV, 1949, p. 447-459, fig. 1-4.

Dupuis (C.). - Notes à propos des Eurygaster (Hémipt. Pentatomoidea, fam. Scutelleridae). Systématique, Biologie, Parasites. L'Entomologiste, 4 (1948), fasc. 5-6, pp. 202-205.

DupuIs (C.). - Contribution à l'étude morphologique des Homoptères. Stades préimaginaux de Ledra aurita (L.). Remarques sur le développement des organes génitaux externes. Feuille des Naturalistes, Bull. des N.P., n. s. 4, fasc. 5-6, pp. $43-47,5$ fig. 
Dupuis (C.). - Observations biologiques sur les parasites d'Hémiptères hétéroptères à Richelieu (I.-et-L.) en 1946, 1947, 1948. Ann. de Parasitologie hum. et comp., 24, $\mathrm{n}^{\circ} 3-4$, pp. 211-242. (= Contribution VII à l'Etude des Phasiinae cimicophages).

Dupuis (C.). - On the « late melanism » of the larval stages of Pentatomidae (Hemiptera heteroptera). Entomologist's Monthly Mag., 85, pp. 229-230, 2 fig.

DupuIs (C.). - Contributions à l'Etude des Phasiinae cimicophages (Diptères Larvaevoridae). VIII : Notes biologiques et de morphologie larvaire sur la sous-tribu Allophorina. Ann. de Parasitologie hum. et comp., 24 (1949), $\mathrm{n}^{\circ} 5-6$, pp. 503-546, $26+3$ fig.

Lagrange (E.). - Essais de chimiothérapie sur la Filaire Icosiella neglecta de la grenouille verte Rana esculenta. Ann. de Parasit., XXIV, 1949, p. 49-53, fig. 1, pl. V.

THÉodoridès (J.). - Considérations sur les Paedurus vésicants (Coléopt. Staphylinidae) et essais de vésication avec des espèces de France. Bull. Soc. Path. Exot., 42, p. 14, 1949.

\section{0}

Jubilé du Professeur Emile Brumpt (21 octobre 1948), une plaquette in- $8^{\circ}$, Paris, imp. Lahure, 1950 , p. 1-54, un portrait frontispice h.t.

BRUMPt (E.). - Docteur Maurice Langeron (3 janvier 1874-27 juin 1950). Ann. de Parasit., XXV, ${ }^{\circ s} 5-6,1950$, p. $363-369,1$ portrait h.t.

BUtTNER (A.). - Première démonstration expérimentale d'un cycle abrégé chez les Trématodes digénétiques. Cas du Plagiorchis brumpti. Ann. de Parasit., XXV, 1950, p. 21-26, fig. 1.

BUTTNER (A.). - Labilité particulière du sexe chez Schistosoma mansoni (Plathelminthe, Trématode). Essai d'interprétation. Ann. de Parasit., XXV, 1950. p. 297-307, fig. I-II.

Butrner (A.). - La progénèse chez les Trématodes digénétiques: Historique (1 ${ }^{\text {re }}$ partie). Ann. de Parasit., XXV, 1950, p. 376-434, fig. 1-26.

Chabaud (A.-G.) et Campana (Y.). - Nouveau parasite, remarquable par l'atrophie de ses organes: Robertdollfusa paradoxa (Nématoda incertae sedis). Ann. de Parasit., XXV, 1950, p. 325-334, fig. 1-4.

Chaвaud (A.-G.). - L'infestation par les Ixodidés provoque-t-elle une immunité chez l'hôte? (2 $2^{\mathrm{e}}$ note). Ann. de Parasit., XXV, 1950, p. 474.

Dollfus (R.-Ph.). - Variations anatomiques chez Distomum cloacicola Max Lühe 1909. Ann. de Parasit., XXV, 1950, p. 141-149, fig. 1-3.

Dupuis (C.). - Sur une prétendue phase subimaginale dans le développement de certains Hémiptères hétéroptères. C. R. Ac. Sciences, 231, pp. 879-880.

LeCLERCQ (M.) et THÉodoridès (J.). - Some parasites of birds and mammals observed recently in France. The Entomologist's Monthly Magazine, $4^{\circ}$ sér., $\mathrm{n}^{\circ} 123\left(\mathrm{n}^{\circ} 1.030\right)$, march 1950, p. 73.

THÉODORIDÈs (J.). - Les Coléoptères parasites accidentels de l'homme et des animaux domestiques (4e note). Ann. de Parasit., XXV, 1950, p. 69-76. 
THÉODORIDÈs (J.). - Experiments on the vesicating properties of species belonging to genus Paederus in France. Coleopt. Bull (U.S.A.), 4, 1950, p. 21-22.

THÉODORIDÈs (J.). - Sur la présence constante de Nématodes larvaires, Diplogasterinae chez des Geotrupinae (Col. Scarabaeoidea) de France. Bull. Soc. Zool. Fr., 74, 1950, p. 277-83.

THÉodoridès (J.). - Remarques sur l'allotrophie chez des Carabiques et d'autres Coléoptères. L'Entomologiste, 1950, 6, p. 79-82.

THÉodoridès (J.). - Observations et remarques sur l'écologie des Nécrophores (Col. Silphidae). Phys. Comp. Ecol., 2, p. 107-25, 8 fig., 1 pl.

THÉodoridès (J.). - Notes diverses sur les Necrophorus (Coléoptères Silphidae). Bull. Inst. Roy. Sc. Nat. Belge, XXVI, 52, 20 p., 6 fig.

\section{1}

Biocca (E.) et Chabaud (A.-G.). Redescription de Seuratum mucronatum (Rud. 1809) (Nematoda). Ann. de Parasit., XXVI, 1951, p. 85-92, fig. 1-4.

Brumpt (E.). - Maurice Langeron (1874-1950). La Presse Médicale, 59ª année, $n^{\circ} 21$, p. 437.

ButTneR (A.). - La progénèse chez les Trématodes digénétiques (suite). Techniques et recherches personnelles. Ann. de Parasit., XXVI, 1951, p. 19-66, fig. 27-45.

ButTNER (A.). - La progénèse chez les Trématodes digénétiques (suite). Recherches personnelles sur deux espèces déjà connues: Ratzia joyeuxi (E. Brumpt 1922) et Pleurogenes medians (Olsson 1876). Ann. de Parasit., XXVI, 1951, p. 138-189, fig. 46-57.

Buttner (A.). - La progénèse chez les Trématodes digénétiques (fin). Etude de quelques métacercaires progénétiques à évolution reconnue et de certaines formes de développement voisines de la progénèse. Conclusions générales. Ann. de Parasit., XXVI, 1951, p. 279-322, fig. 58-68.

Dollfus (R.-Ph.). - Sur Distomum polyoon O. von Linstow, parasite mal connu du Ralliforme Gallinula chloropus (L.). Ann. de Parasit., XXVI, 1951, p. $128-131$, fig. 1 .

Dollfus (R.-Ph.). - Miscellanea helminthologica maroccana I. Quelques Trématodes, Cestodes et Acanthocéphales. Arch. Inst. Pasteur Maroc. Casablanca, IV, cahier 3, p. 104-229, fig. 1-62.

Dupuis (C.). - Allochromie liée aux modifications du métabolisme chez des Hémiptères des genres Dolycoris et Carpocoris. C. R. Ac. Sciences, 232, pp. 262-264.

DupuIs (C.). - Les Insectes parasites entomophages. La Feuille des Naturalistes, Bull. des N.P., n. s. 6, 1951, fasc. 5-6, pp. 45-54.

Dupuis (C.). - Note préliminaire sur Strawinskiomyia (n. g.) costata (Panzer 1801). $\mathrm{XII}^{\circ}$ Contribution à l'Etude des Phasiinae cimicophages (Diptères Larvaevoridae). Bull. Soc. Zool. Fr., 76 (1951), n 3, pp. 129-137, 1 fig.

Dupuis (C.). - Sur une larve d'Hyménoptère braconide, parasite de Pentatomides (Hem. heteroptera). IX th Int. Congr. Entom. Abstracts of papers, Amsterdam, 1951, pp. vi-2-vI-3. 
Dupuis (C.). - Id. Trans. IX th Int. Congr. Ent., Amsterdam, 1951, 1 (1952), pp. 539-540.

\section{2}

Buttner (A.). - Cycle évolutif de Ratzia joyeuxi (E. Brumpt, 1922) (Trematoda, Opisthorchiidae). Ann. de Parasit., XXVII, 1952, $\mathrm{n}^{\circ s}$ 1-3 (In Memoriam E. Brumpt), p. 105-142, fig. 1-18.

Dollfus (R.-Ph.). - Quelques Oxyuroidea de Myriapodes. Ann. de Parasit., XXVII, 1952, p. 143-236.

Dollfus (R.-Ph.). - Premier supplément (novembre 1941-décembre 1952) aux Titres et travaux scientifiques de Robert-Ph. Dollfus. Paris, déc. 1952, $42 \mathrm{p}$.

Dupuis (C.). - Notes, remarques et observations diverses sur les Hémiptères. Première série : notes I-IV. Feuille des Naturalistes, Bull. des N.P., n. s. 7, fasc. 1-2, pp. 1-4.

DupuIs (C.). - Contributions XIII et XIV à l'Etude des Phasiinae cimicophages (Diptères Larvaevoridae). XIII : Notes synonymiques et systématiques. XIV: Hôtes inédits et localités nouvelles. Ann. de Parasitologie hum. et comp., 27 (1952), fasc. 1-3 (fasc. In memoriam, Prof. E. BRUMPT), pp. 329-338.

Dupuis (C.). - Notes, remarques et observations diverses sur les Hémiptères. Deuxième série : note V: Ceresa bubalus (F.) en Touraine (Membracidae). Feuille des Naturalistes, Bull. des N.P., n. s. 7, fasc. 7-8, p. 73.

Galliard (H.). - Le Professeur Emile Brumpt (1877-1951). Ann. de Parasit., XXVII, 1952, $\mathrm{n}^{\circ s}$ 1-3 (In Memoriam E. Brumpt), p. 5-46, 2 portraits h.t.

\section{3}

Buttner (A.). - Un curieux cas de phorésie: transport de 21 Ancylus fluviatilis Müller par un Dytique femelle (Dysticus marginalis L.) et possibilité de diffusion des cercaires parasites de ces Mollusques pulmonés. Ann. de Parasit., XXVIII, 1953, p. 452-453, fig. 1.

Buttner (A.). - Valeur évolutive de la progénèse chez les Trématodes Digénétiques. Thapar Commemoration Volume, 1953.

ButTNER (A.). - Sur l'aptitude de certains Rongeurs sauvages à devenir réservoirs de virus de la bilharziose intestinale humaine. Bull. Soc. Path. Exot., 46, 1953, p. 696-700.

Dollfus (R.-Ph.) et BuTrNer (A.). - Localisations anormales de Metorchis xanthosomus (Creplin 1846) chez un canard domestique (canard d'Inde). Ann. de Parasit., XXVIII, 1953, p. 450-452, fig. I-II.

Dupurs (C.). - Contributions à l'étude des Phasiinae cimicophages (Diptera Larvaevoridae). XV: Données sur les Leucostomatina, et, en particulier, Leucostoma analis (Meigen) s. str. Ann. Parasitologie hum. et comp., 28. $\mathrm{n}^{\circ \mathrm{s}} 1-2$, p. $64-97,20$ fig. + pl. I-II.

DupuIs (C.). - Notes, remarques et observations diverses sur les Hémiptères. Troisième série: notes VI-VIII. Cahiers des Naturalistes, Bull. des N.P., n. s. 8 , fasc. $3-4$, p. $25-29$. 
Dupurs (C.). - Les Rhopalidae de la Faune française (Hemiptera-Heteroptera). Caractères généraux. Tableaux de détermination. Données monographiques sommaires. Cahiers des Naturalistes, Bull. des N.P., n. s. 8, fasc. 7-8, p. $67-82$, 42 fig.

\section{4}

ARVy (L.) et Butrner (A.). - Données sur le cycle évolutif de Diplostomulum phoxini (Faust 1918) (Trematoda, Diplostomidae), C.R. Ac. Sciences, 239 , p. $1085-1087$.

Сhabaud (A.G.). - Sur le cycle évolutif des Spiruridés et de Nématodes ayant une biologie comparable. Valeur systématique des caractères biologiques. Ann. de Parasit., XXIX, 1-2, 1954, p. 42-88, fig. 1-20.

Dollfus (R.-Ph.). - Miscellanea Helminthologica maroccana, XIII. Deux Dicrocœliinae d'Oiseaux Passériformes du Maroc. Discussion de quelques genres de Dicrocaliinae d'homéothermes. Arch. Inst. Pasteur Maroc, Casablanca, IV, cahier 9, 15-9-1954, p. 583-602, fig. 19-24.

Dupurs (C.). - Observations et expériences pour l'interprétation d'infestations naturelles d'imagos mâles d'Ectophasia par des larves I de leur propre espèce (Diptera Larvaevoridae, subfam. Phasiinae). (Contribution à l'étude des Phasiinae cimicophages, XVII). C.R. Ac. Sciences, 239, 1954, p. 836-838.

THÉoDoridès (J.). - Contribution à l'étude des parasites et phorétiques de Coléoptères terrestres. L'Année Biol., 30, 145-167, 1954.

\section{5}

Arvy (L.) et Butrner (A.). - Cycle évolutif de Diplostomulum phoxini (Faust 1918) (Diplostomatidae). Bull. Soc. Zool. France, LXXX, 1955, p. 104.

Bourcart (N.). - Technique d'isolement d'une souche neuve d'Helobdella algira, à partir d'exemplaires parasités par des Trypanosomes d'Amphibiens. Ann. de Parasit., XXX, 1955, p. 504.

Butrner (A.). - Les Distomes progénétiques sont-ils des préadultes ou des adultes véritables? Valeur évolutive de la progénèse chez les Digenea. C.R. Séa. Soc. Biol., CXLIX, 1955, 267-272.

ButTNer (A.) et BourCaRT (N.). - Sur certaines particularités biologiques d'un Trypanosome de la Grenouille verte, Trypanosoma inopinatum Sergent, 1904. Ann. de Parasit., XXX, 1955, p. 431-445.

Golvan (Y.-J.) et ThÉodoridès (J.). - Le Hérisson (Erinaceus europaeus L.), prédateur des larves d'un Culicidé : Aedes rusticus Rossi 1790. Ann. de Parasit., XXX, 1955, p. 421-422.

Leclerce (Marcel). - Tabanidae (Dipt.) de France. Bull. Ann. Soc. Roy. Ent. Belg., 91, III-IV, 1955, p. 76-83.

THÉodoridès (J.). - Contribution à l'étude des parasites et phorétiques de Coléoptères terrestres, 1 vol., 310 p., Actual. Scient. Ind., Hermann, Suppl. Vie et Milieu, $\mathrm{n}^{\circ} 4,1955$. 


\section{6}

Liste des travaux de la Station expérimentale de Richelieu (Indre-et-Loire). Annales de l'Université de Paris, XXVI, $\mathrm{n}^{\circ}$ 1, janv.-mars 1956, p. 103-110.

Chabaud (A.-G.) et Golvan (Y.-J.). - Nouvelle Filaire parasite des Grives en France. Ann. de Parasit., XXX, p. 405-413, fig. 1-3.

Dollfus (R.-Ph.). - Distoma arenula F. C. H. Creplin 1825, Distome peu connu, trouvé chez la Poule d'eau, Gallinula chloropus (L.), à Richelieu (I.-et-L.). Ann. de Parasit., XXXI, 1956, p. 182-188, fig. 1.

Dupuis (C.). - Contributions à l'étude des Phasiinae cimicophages (Diptera Larvaevoridae), XVIII. Une espèce méconnue, nouvelle pour la faune française, Hyalomyia barbifrons (Girschner 1887). Cahiers des Naturalistes, Bull. des N.P., n. s. 11, fasc. 4 (1955), 1956, p. 89-93.

DupuIs (C.). - Titres et travaux scientifiques de Claude Dupuis, une brochure hors-commerce, polycopiée, 32 p. $(21 \times 27 \mathrm{~cm}$.), mars 1956.

Golvan (Y.-J.). - Acanthocéphales d'Oiseaux (Troisième note). Révision des espèces européennes de la sous-famille des Plagiorhynchinae A. Meyer 1931 (Polymorphidae). Ann. de Parasit., XXXI, 1956, p. 350-384, fig. $1+$ pl. in texte I-IV.

\section{7}

Deblock (S.), CAPron (A.) et Biguet (J.). - Contribution à la connaissance d'Asymphilodora tincae (Modeer 1790). Ann. de Parasit., XXXII, 1957, p. 208-218, fig. 1-10.

Dollfus (R.-Ph.). - Miscellanea Helminthologica maroccana, XX. Contribution à la connaissance des Nematotaenia. Arch. Inst. Pasteur Maroc, Casablanca, V, cahier 7, p. 301-328, fig. 1-16.

Dollfus (R.-Ph.). - Miscellanea Helminthologica maroccana, XXI. Quelques Cestodes d'Otidiformes, principalement d'Afrique du Nord. Répartition géographique des Cestodes d'Otidiformes. Arch. Inst. Pasteur Maroc, Casablanca, V, cahier 7, p. 329-402, fig. 1-50.

Dollfus (R.-Ph.). - Miscellanea Helminthologica maroccana, XXIII. Nématodes d'Otidiformes (en collaboration avec Alain-G. Chabaud). Arch. Inst. Pasteur Maroc, Casablanca, V, cahier 7, p. 408-446, fig. 1-2.

Dollfus (R.-Ph.). - Sur trois Distomes (Telorchis, Opisthioglyphe, Astiotrema) de Couleuvres du genre Natrix Laurenti 1768. Ann. de Parasit., XXXII, $\mathrm{N}^{\circ s} 1-2,1957$, p. 41-55, fig. 1-6.

Dupurs (C.). - Comptes rendus des principales excursions des Naturalistes parisiens en 1956. Cahiers des Naturalistes, Bull. des N.P., n. s. 12, fasc. 4 (1956), 1957, p. 101-125 (en collaboration avec Daniel RAPILlY).

Dupuis (C.). - Contributions à l'étude des Phasiinae cimicophages (Diptera Larvaevoridae). XIX. Etude de Cylindromyia pilipes (Lw.) s. str. Cahiers des Naturalistes, Bull. des N.P., n. s. 13, fasc. 1, 1957, p. 9-22.

Dupuis (C.). - Développement expérimental de larves de Phasiinae (Diptera Lar- 
vaevoridae) chez un hôte non spécifique. C.R. Séances Acad. Sc., 245, $\mathrm{n}^{\circ} 18,1957$, p. 1579-1580. (Contributions à l'étude des Phasiinae cimicophages, XX).

Dupuis (C.). - Contributions à l'étude des Phasiinae cimicophages (Diptera Laraevoridae), XXI. Notes taxonomiques et biologiques diverses. Cahiers des Naturalistes, Bull. des N.P., n. s. 13, fasc. 3, 1957, p. 71-79.

LeClerce (M.). - Révision systématique et biogéographique des Tabanidae (Dipt.) de France, II. Ann. de Parasit., XXXII, p. 398-43i.

\section{8}

Chabaud (A.-G.) et Mahon (J.). - Cycle évolutif du Nématode Spirura talpae (Gmelin, 1790). C.R. Soc. Biol., CLII, p. 474-476.

Dollfus (R.-Ph.). - Les Dicrocoelinae d'Oiseaux décrits ou mentionnés en 1900 par Alcide Railliet et quelques autres. Ann. de Parasit., XXXII, $\mathrm{n}^{\circ} 4$ (1957), 1958, p. 369-384, fig. 1-16 (incl. 1 dépliant h. t.).

Dollfus (R.-Ph.). - Opisthioglyphe endoloba (F. Dujardin 1845) est une espèce distincte d'Opisthioglyphe ranae (Froelich 1791) A. Looss 1907. Ann. de Parasit., XXXIII, 1958, p. 218-226, fig. 1-6.

Dupurs (C.). - Titres et travaux scientifiques de Claude Dupuis. Premier Supplément, une brochure hors-commerce, polycopiée, 8 p. $(21 \times 27 \mathrm{~cm}$.), déc. 1958.

Roman (E.). - Contribution à la répartition en France de la famille des Culicidae. Gîtes larvaires particuliers de quelques-uns d'entre eux. Ann. Parasit., XXXIII, 1958, p. 115-130.

\section{9}

Buttner (A.) et VACHeR (C.). - Evolution d'un Plagiorchis s'enkystant chez Gammarus pulex L. et identifié à Plagiorchis cirratus (Rud. 1802) (Trematoda Plagiorchiidae). C.R. Séa. Soc. Biol., CLIII, 1959, p. 1712-1718.

ButTNER (A.). - Observations sur le genre Schizotrypanum Chagas, 1909. Anais do Congresso Internacional sobre Doença de Chagas, Rio-de-Janeiro, 1959.

Chabaud (A.-G.) et Campana-Rouget (Y.). - Hyalomma anatolicum Koch 1844 chez un Oiseau à Richelieu (Indre-et-Loire). Ann. Parasit., N. et. I., 34, p. 728-729.

Dollfus (R.-Ph.). - Deuxième Supplément (décembre 1952-janvier 1959) aux Titres et travaux scientifiques de Robert-Ph. Dollfus, Paris, janv. 1959, 40 p. (tirage au ronéo).

Dollfus (R.-Ph.). - Sur un Distome de la famille des Coïtocaecidae pourvu d'un receptaculum seminis. Bull. Soc. Zool. de France, LXXXIII, 1959, p. 370376, fig. 1-3. 
1960

Buttner (A.) et VACHer (C.). - Recherches sur le développement de Plagiorchis (Multiglandularis) cirratus (Rudolphi, 1802). I. Etude du cycle évolutif. Ann. de Parasit., XXXV, 1960, p. 268-281, fig. 1-4.

Doby (J.-M.) et Rault (B.). - Présence d'Aedes excrucians Walker 1856 à Richelieu (Indre-et-Loire). Observations écologiques concernant cette espèce. Ann. de Parasit., XXXV, p. 619-621.

DoBY (J.-M.) et RAulT (B.). - Complément à l'étude des caractères morphologiques différentiels de Culex pipiens Linné 1758 et de Culex torrentium Martini 1924. Cahiers des Naturalistes, Bull. des N.P., n. s. 16, fasc. 4, 1960, paru 6-7-1961, p. 113-121, fig. I-III.

Dollfus (R.-Ph.). - Sur un Taenia (Multiceps) du renard, Vulpes vulpes (L.), discussion de son identification spécifique. Parassitologia, Roma, vol. 1, $\mathrm{n}^{\circ} 2$ (oct. 1959), janvier 1960, p. 143-165, fig. 1-23.

Dollfus (R.-Ph.). - Recherches expérimentales sur Nicolla gallica (R.-Ph. Dollfus 1941) R.-Ph. Dollfus 1958, sa cercaire cotylicerque et sa métacercaire progénétique. Observations sur la famille des Coïtocaecidae Y. Osaki 1918, s.-f. Coïtocaecinae F. Poche 1926 (Trematoda-Podocotyloidea) et sur les cercaires cotylicerques d'eau douce et marines. Ann. Parasit., XXXIV, 1959, p. 595-624, et XXXV, 1960, p. 65-117, fig. 1-39.

Dollfus (R.-Ph.). - Recherches sur l'identification de Plagiorchis (Multiglandularis) cirratus (Rud. 1802). II. Description et identification. Ann. Parasit., XXXV, 1960, p. 282-291, fig. 1.

Dupuis (C.). - Contributions à l'étude des Phasiinae cimicophages (Diptera, Larvaevoridae). XXIV. Les Gymnosoma ouest-paléarctiques (à l'exclusion du groupe de costata Pz.). Cahiers des Naturalistes, Bull. des N.P., n. s. 16, fasc. 3 (1960), 1961, p. 69-75.

LE VAN HoA. - Redescription de quelques Capillaria peu connus récoltés à Richelieu (Indre-et-Loire). Ann. de Parasit., XXXV, 1960, p. 594-606, fig. 1-4.

PoJMANSKa (Teresa) et RYBicka (Krystyna). - Wipomnicnia zpobytu w nicktorych francus kich osrodkack parazytologieznych. Wiadomósci Parazytologiczne, Wroclaw, VI, $\mathrm{n}^{\circ} 23$, p. 242-248, fig. 1-2.

THÉodoridès (J.). - Parasites et Phorétiques de Coléoptères et de Myriapodes de Richelieu (Indre-et-Loire). Ann. de Parasit., XXXV, p. 488-503, fig. 1-4.

Chabaud (A.-G.). - Titres et travaux scientifiques, une brochure in- $4^{\circ}$, Paris, 49 p., pl. I-VII.

\section{1}

Gretillat (Simon). - Description de deux nouvelles espèces de Rhinonyssidae (Acarina, Mesostigmata): Rallinyssus strandtmanni n. sp. et Larinyssus petiti n. sp. Vie et milieu, t. XII (1961), fasc. 1, p. 151-160, fig. 1-5.

Jurewicz (Ewa). - Remarks on the life cycle of Plagiorchis elegans (Rud. 1802) (Trematoda, Plagiorchiidae) and the problem of revision of the genus Plagiorchis Lühe 1899. Wiadomôsci Parazytologiczne, Olsztyn 9-6-1961. Nr. 2. Supplementum, p. 191-194. 